\title{
Lutte contre la maladie du bayoud par solarisation et fumigation du sol. Une expérimentation dans les palmeraies du Maroc
}

\author{
Adil Essarioui ${ }^{1, *}$ et Moulay Hassan Sedra ${ }^{2,3}$ \\ 1 Inra, Errachidia, Maroc \\ 2 Inra, Marrakech, Maroc \\ ${ }^{3}$ FAO, Rabat, Maroc
}

\begin{abstract}
Résumé - Le bayoud, maladie vasculaire causée par Fusarium oxysporum f. sp. albedinis, est la menace principale pour le palmier dattier au Maroc. L'effet de la solarisation et de fumigation par le metam sodium, seules ou combinées, sur les champignons telluriques en général et les Fusarium spp. en particulier, a été étudié en milieu réel. Entre 0 et $40 \mathrm{~cm}$ de profondeur, tous les traitements ont éliminé plus de $80 \%$ de la flore fongique totale et $90 \%$ des germes de Fusarium spp. Dans la couche $40-60 \mathrm{~cm}$, la combinaison de la solarisation et du metam sodium à doses réduites est nécessaire pour éliminer les Fusarium. Le couplage de la solarisation et de la fumigation pourrait être envisagé en cas d'apparition de la maladie du bayoud dans les nouvelles plantations de palmier dattier.
\end{abstract}

Mots clés : solarisation / metam sodium / bayoud / palmier dattier

\begin{abstract}
Management of bayoud disease using soil solarization and fumigation. An experiment in date palm plantations in Morocco. Bayoud, a vascular disease incited by Fusarium oxysporum f. sp.albedinis, is the principal threat to date palm in Morocco. The effect of soil solarization and/or fumigation using metam sodium, on survival of total fungi and Fusarium spp., was investigated in a field trial. At $40 \mathrm{~cm}$ depth, all treatments eliminated more than $80 \%$ of total fungi and $90 \%$ of Fusarium spp. At $40-60 \mathrm{~cm}$ depth, the combination of reduced doses of metam sodium with soil solarization resulted in the greatest impact on Fusarium survival. The combination of soil solarization with fumigation offers significant potential for managing the possible spread of bayoud in modern Moroccan palm orchards.
\end{abstract}

Keywords: soil solarization / metam sodium / bayoud / date palm

\section{Introduction}

Le palmier dattier est la culture principale au Sud-est du Maroc. La palmeraie marocaine, auparavant constituée principalement de plantations traditionnelles le long des oueds et autour de points d'eau éparpillés du Sud-est au Sud-ouest, connaît actuellement une nouvelle dynamique. Pour atteindre ses objectifs de modernisation du secteur phoenicicole et d'intensification de la production dattière, l'État subventionne la création de nouvelles plantations. Grâce à cette politique, des milliers d'hectares de palmiers dattiers ont été plantés et de grands projets modernes ont vu le jour.

Le bayoud, fusariose vasculaire du palmier dattier causée par Fusarium oxysporumf.sp. albedinis, représente une importante menace pour ces investissements. Depuis son

\footnotetext{
*Auteur de correspondance : essar002@umn.edu
}

identification il y a plus d'un siècle, la maladie sévit dans les oasis traditionnelles marocaines, y causant des pertes économiquement considérables et une érosion génétique qui menace l'équilibre fragile de l'écosystème oasien. Les efforts de lutte contre cette épidémie se sont axés sur la lutte génétique. Les résultats ont été concluants et plusieurs variétés et clones résistants ont été sélectionnés et utilisés dans de multiples programmes de reconstitution des zones dévastées par la maladie dans les palmeraies traditionnelles (Sedra, 2005). En revanche, les nouvelles plantations sont basées essentiellement sur la culture de variétés à haute valeur commerciale, comme «Majhoul», très sensibles à la maladie. Elles sont établies dans des zones proches des palmeraies traditionnelles, sur des terrains jamais cultivés et très probablement indemnes de l'agent pathogène. Mais la proximité des nouvelles plantations avec des foyers actifs de la maladie, la monoculture, l'uniformité génétique et l'intensification augmentent les risques d'attaque par la maladie du bayoud. 
La solarisation du sol (couverture par un film plastique transparent et hermétique) est une technique efficace pour la baisse de la densité des agents pathogènes telluriques et la réduction des maladies (Porras et al., 2007; Funahashi et Parke, 2016). La solarisation du sol a en particulier été rapportée comme moyen de traitement de pré-plantation pour lutter contre de nombreuses fusarioses vasculaires, aussi bien chez des plantes annuelles, comme la tomate (Barakat et AlMasri, 2012) ou le melon (Tamietti et Valentino, 2006), que pérennes, comme le bananier (Ploetz, 2015) ou le palmier à huile (Flood, 2006). Le rôle potentiel de la solarisation dans la lutte contre l'agent du bayoud a été mis en évidence dans des travaux antérieurs (Essarioui, 2006). La solarisation est très active contre les espèces du genre Fusarium, auquel appartient l'agent causal de la fusariose vasculaire du palmier dattier. Cependant, son effet décroît rapidement en profondeur.

La fumigation du sol pourrait aussi contribuer à diminuer le potentiel infectieux du sol. C'est la principale technique utilisée pour éliminer des agents pathogènes telluriques (DeVay, 1998; Hwang et al., 2014). Depuis l'interdiction de l'usage du bromure de méthyle pour ses effets sur la couche d'ozone (protocole de Montréal), des études visant le développement d'alternatives ont été menées (Duniway, 2002). Le metam sodium est l'un des produits ayant montré une bonne aptitude à contrôler les pathogènes telluriques (Hamm et al., 2003; Shachaf et al., 2007; Xie et al., 2015).

La combinaison de plusieurs techniques peut être un outil important de la lutte intégrée contre les parasites (Katan, 1996). L'association de la fumigation à des doses réduites avec la solarisation ou avec d'autres moyens de lutte améliore l'efficacité des traitements (Shachaf et al., 2007). En effet, l'affaiblissement des agents pathogènes par des doses souslétales de produits chimiques accroît leur fragilité et les rend plus vulnérables aux autres agents biotiques et abiotiques du sol, tels que les antagonistes et la chaleur.

La présente étude a été conduite dans l'objectif d'évaluer les effets de la solarisation et de la fumigation par le metam sodium, seules ou combinées, sur la survie des champignons telluriques en général et des Fusarium spp. en particulier.

\section{Matériel et méthodes}

\subsection{Choix des parcelles}

L'étude a été menée dans une parcelle naturellement infestée par l'agent causal du bayoud sur le domaine expérimental de l'Inra à Zagora (Maroc). Les palmiers de cette parcelle ont été totalement détruits par la maladie du bayoud durant les années 1980 .

\subsection{Dispositif expérimental}

Le dispositif expérimental était une randomisation totale de 5 traitements répétés 3 fois (Fig. 1). Le sol de la parcelle de l'essai a subi un labour croisé à $25 \mathrm{~cm}$ de profondeur avec une charrue à socs puis un covercropage croisé pour casser les mottes. Quinze petites parcelles $(2 \mathrm{~m} \times 2 \mathrm{~m})$, espacées de $2 \mathrm{~m}$ en tous sens, ont été ensuite aménagées (nivellement et mise en
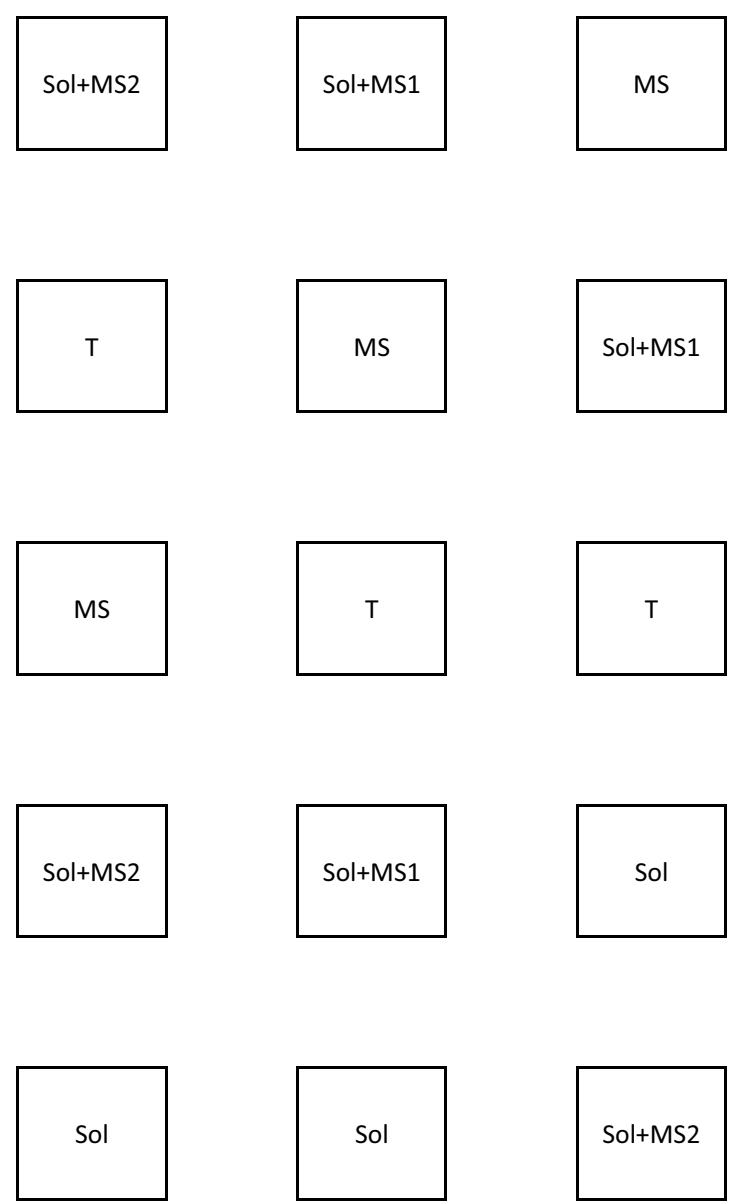

Fig. 1. Schéma du dispositif expérimental en randomisation totale de 5 traitements $\times 3$ répétitions. Les carreaux représentent les parcelles élémentaires $(2 \mathrm{~m} \times 2 \mathrm{~m})$ espacées de $2 \mathrm{~m}$ en tous sens. T: témoin; Sol: solarisation; MS : metam sodium $\left(61,2 \mathrm{~g} / \mathrm{m}^{2}\right)$; Sol + MS1 : solarisation et metam sodium $\left(30,6 \mathrm{~g} / \mathrm{m}^{2}\right) ; \mathrm{Sol}+\mathrm{MS} 1$ : solarisation et metam sodium $\left(40,8 \mathrm{~g} / \mathrm{m}^{2}\right)$.

Fig. 1. Layout of the experimental complete randomized design (5 treatments $\times 3$ replicates). Squares represent experimental units (plots, $2 m \times 2 m) 2 m$ apart in all directions. T: control; Sol: solarization; MS: metam sodium $\left(61.2 \mathrm{~g} / \mathrm{m}^{2}\right)$; Sol + MS1: solarization and metam sodium $\left(30.6 \mathrm{~g} / \mathrm{m}^{2}\right) ; \mathrm{Sol}+\mathrm{MS} 1$ : solarization and metam sodium $\left(40.8 \mathrm{~g} / \mathrm{m}^{2}\right)$.

cuvette) pour constituer les parcelles élémentaires de chaque traitement ( 5 traitements $\times 3$ répétitions).

\subsection{Mise en place de l'essai et traitements}

L'étude a été réalisée durant les mois de juillet et août 2010. La spécialité commerciale de metam sodium utilisée dans l'essai était le NEMASOL 510 ( $510 \mathrm{~g} / \mathrm{L}$ de matière active). Le film plastique utilisé est un polyéthylène transparent ayant une épaisseur de 40 microns.

Les traitements sont les suivants:

- solarisation (Sol): les parcelles concernées ont été irriguées jusqu'à la saturation puis couvertes hermétiquement avec le film plastique dont les extrémités ont été enterrées ; 
- fumigation au metam sodium (MS) à la dose de $120 \mathrm{~mL} / \mathrm{m}^{2}$ de produit commercial, dose prescrite par le fabricant (soit $61,2 \mathrm{~g} / \mathrm{m}^{2}$ ) : le produit préalablement dilué dans $10 \mathrm{~L}$ d'eau a été déversé dans les parcelles qui ont été irriguées immédiatement pour entraîner le fumigant en profondeur; - combinaison de la solarisation et du metam sodium $(\mathrm{Sol}+\mathrm{MS} 1)$ à la dose de $60 \mathrm{~mL} / \mathrm{m}^{2}$ de produit commercial (soit $30,6 \mathrm{~g} / \mathrm{m}^{2}$ ): le sol a ici été couvert avec le film plastique immédiatement après le traitement par le metam sodium;

- combinaison de la solarisation et du metam sodium $(\mathrm{Sol}+\mathrm{MS} 2)$ à la dose de $80 \mathrm{~mL} / \mathrm{m}^{2}$ de produit commercial (soit 40,8 g/m²); même mise en œuvre que pour Sol + MS1 ;

- témoin (T): les parcelles témoins ont seulement été irriguées jusqu'à la saturation.

Une expérience préalable avait permis d'évaluer la quantité d'eau nécessaire pour humecter le sol à plus de $60 \mathrm{~cm}$ de profondeur.

\subsection{Prélèvement des échantillons}

Le prélèvement des échantillons a été fait à l'aide d'une tarière après huit semaines de traitement. Dans chaque unité expérimentale, les prélèvements ont été faits dans trois points choisis au hasard et à trois niveaux de profondeur, à savoir : 0$20 \mathrm{~cm}, 20-40 \mathrm{~cm}$ et $40-60 \mathrm{~cm}$. Les échantillons venant de la même parcelle et du même niveau de profondeur ont ensuite été mélangés et homogénéisés puis mis dans des sachets en papier kraft et ramenés au laboratoire pour l'analyse microbiologique (échantillons composites).

\subsection{Analyse microbiologique}

L'analyse microbiologique avait pour objectif l'évaluation de l'effet des différents traitements sur la densité de la flore fongique totale en général et de la flore fusarienne en particulier, en utilisant le principe classique des suspensions dilutions. Chaque échantillon a subi une série d'opérations successives (séchage, broyage et tamisages) selon la technique utilisée par Sedra (1993). Les particules de terre de l'échantillon final avaient un diamètre inférieur ou égal à $80 \mu \mathrm{m}$. Trois sous-échantillons de $10 \mathrm{mg}$ chacun ont été prélevés dans chaque échantillon composite de sol, homogénéisés, et dilués dans $30 \mathrm{~mL}$ d'eau distillée stérile. Les dilutions réalisées étaient $10^{-3}, 10^{-2}$ et $10^{-1}$ pour les souséchantillons venant respectivement des profondeurs $0-20 \mathrm{~cm}$, $20-40 \mathrm{~cm}$ et $40-60 \mathrm{~cm}$. Le dénombrement de chaque type de microorganisme a été effectué sur dix boites de Pétri pour chaque sous-échantillon composite de sol. Les boites d'analyse ensemencées ont été incubées à la température ambiante à l'obscurité $(16 \mathrm{~h})$ puis sous éclairage continu (lumière fluorescente) selon la technique utilisée par Sedra (1993). La flore fongique totale a été révélée sur le milieu de culture Gzapeck acidifié par l'acide chlorhydrique $(\mathrm{pH}=4)$ et la flore fusarienne sur le milieu de culture sélectif Komada (1975). Les résultats de l'analyse microbiologique sont exprimés en nombre d'unités formant des colonies, traduisant le nombre de propagules par gramme de sol.

\subsection{Analyse statistique}

L'analyse statistique a été réalisée à l'aide du logiciel SPSS. Une analyse de la variance à un seul critère de classification avec un seuil de signification $p=0,05$ a été conduite pour tester l'effet des traitements. Les conditions d'application de l'analyse de variance, notamment la normalité des résidus et l'égalité des variances, ont été vérifiées. Le test de Newman-Keuls a été utilisé pour la séparation des groupes de moyennes statistiquement homogènes.

\section{Résultats}

\subsection{Effet sur la flore fongique totale}

Comparés au témoin, tous les traitements entraînent des chutes remarquables dans les populations de la flore fongique totale dans le sol des différentes parcelles traitées. Mais l'efficacité de ces traitements varie en fonction de la profondeur du sol.

Au niveau de la couche $0-20 \mathrm{~cm}$ (Fig. 2A), l'efficacité des différents traitements est maximale. L'effet de la combinaison du metam sodium avec la solarisation est légèrement supérieur à ceux des deux traitements séparés. Vers la fin de l'expérience, les deux combinaisons sont venues à bout de $100 \%$ des champignons telluriques. Le metam sodium a éliminé $98 \%$ de cette catégorie de microflore, alors que la solarisation en a éliminé seulement $86 \%$.

L'efficacité dans la couche médiane $(20-40 \mathrm{~cm})$ est similaire à celle dans la couche supérieure (Fig. 2B). Cependant, l'avantage de la combinaison des deux traitements est plus net. En effet, seule la combinaison Sol + MS2 a anéanti tous les germes de champignons. L'autre combinaison a montré une efficacité comparable au traitement au seul metam sodium. Ils ont, tous les deux, détruit $97 \%$ des champignons. La solarisation est beaucoup moins efficace, avec un taux de réussite de $84 \%$.

En profondeur (couche $40-60 \mathrm{~cm}$ ), les différents traitements, et notamment la solarisation, perdent de l'effet (Fig. 2C). Les efficacités étaient ainsi respectivement de $85 \%, 76 \%, 68 \%$ et $62 \%$ pour la combinaison Sol + MS2, Sol + MS1, le metam sodium et la solarisation.

\subsection{Effet sur la flore fusarienne}

L'action du produit chimique et de la solarisation sur la flore fusarienne est similaire à celle sur la flore fongique totale, même si les pourcentages de réduction de la flore fusarienne sont plutôt meilleurs dans la majorité des cas.

La couche supérieure (0-20) est la plus affectée par les traitements (Fig. 2D). C'est aussi la combinaison de la solarisation et du metam sodium qui donne le meilleur résultat, avec $100 \%$ de réussite. Séparément, les deux moyens de lutte entraînent une réduction de $97 \%$.

Un peu plus profond, dans la strate $20-40 \mathrm{~cm}$, tous les traitements maintiennent leur pression sur les Fusarium du sol (Fig. 2E). Les taux d'efficacité y varient entre $94 \%$ dans les parcelles solarisées et $100 \%$ grâce à la combinaison Sol+ MS2.

En profondeur, dans la couche 40-60, les germes sont plus à l'abri de l'action létale des traitements (Fig. 2F). Les deux 
FFT: profondeur $0-20 \mathrm{~cm}$

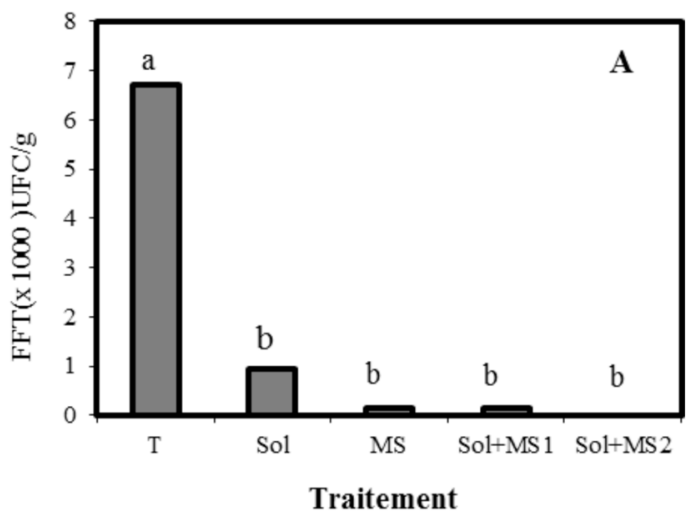

FFT: profondeur $20-40 \mathrm{~cm}$

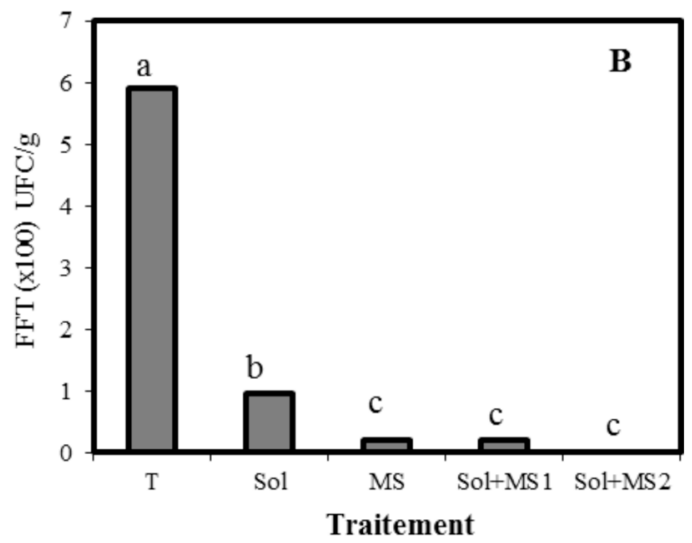

FFT: profondeur $40-60 \mathrm{~cm}$

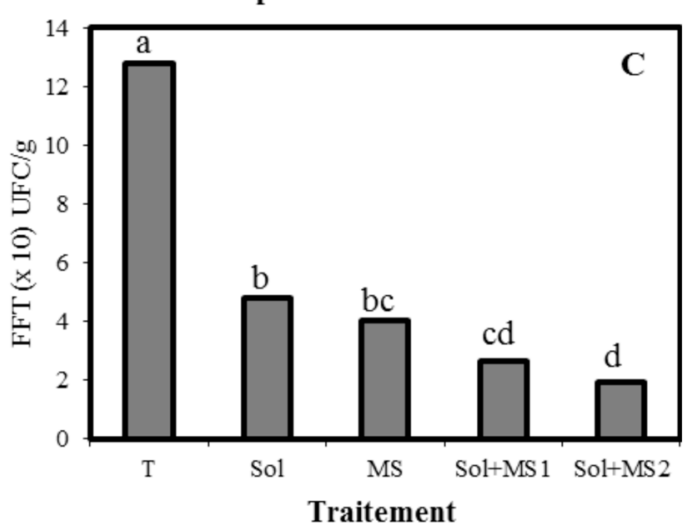

FF.: profondeur $0-20 \mathrm{~cm}$

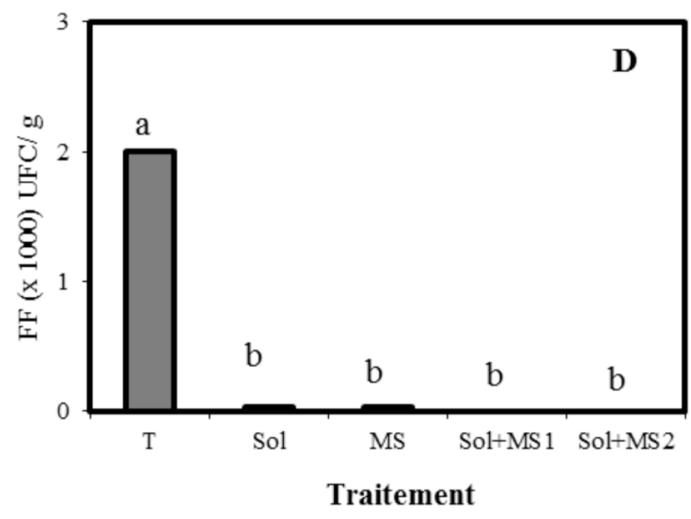

FF: profondeur $20-40 \mathrm{~cm}$

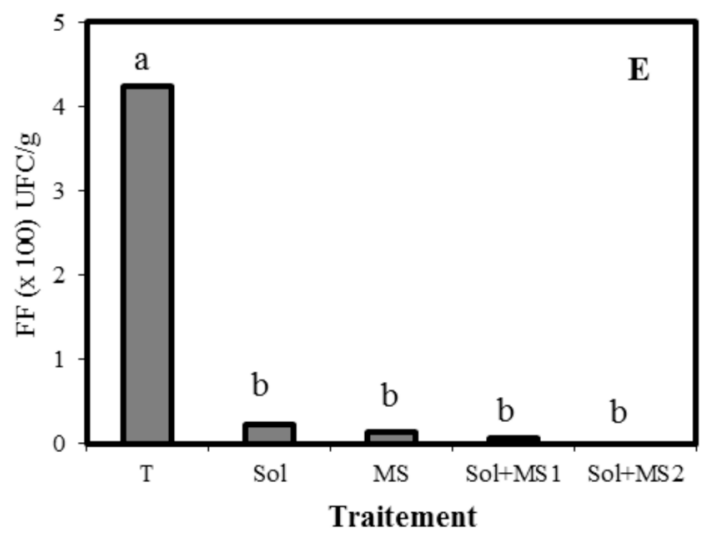

FF: profondeur $40-60 \mathrm{~cm}$

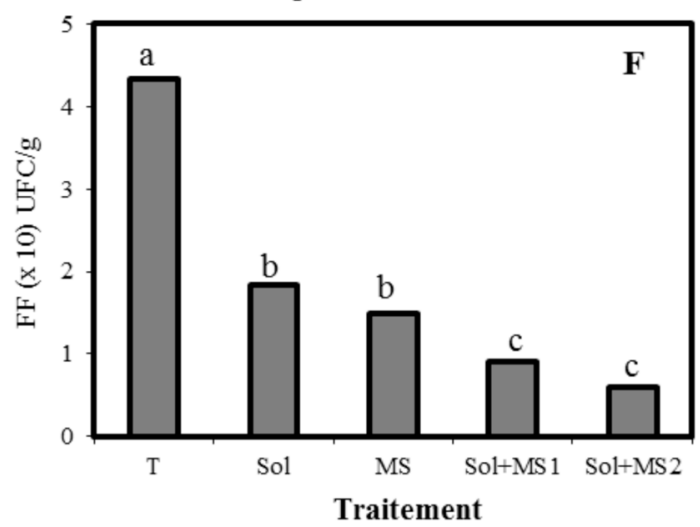

Fig. 2. Effet des traitements sur la densité de la flore fongique totale (FFT; panels A, B et C) et de la flore fusarienne (FF ; panels D, E et F) à différentes profondeurs du sol. T : témoin ; Sol : solarisation; MS : metam sodium $\left(61,2 \mathrm{~g} / \mathrm{m}^{2}\right)$; Sol + MS1 : solarisation et metam sodium (30,6 g/ $\left.\mathrm{m}^{2}\right)$; Sol + MS2 : solarisation et Metam sodium $\left(40,8 \mathrm{~g} / \mathrm{m}^{2}\right)$. Les traitements suivis de la même lettre ne sont pas significativement différents pour $p=0,05$ (test de Newman-keuls).

Fig. 2. Treatment effects on the density of total fungi (FFT; panels $A, B$ and $C$ ) and Fusarium flora (FF; panels D, E and F) at different soil depths. T: control; Sol: solarization; MS: metam sodium $\left(61.2 \mathrm{~g} / \mathrm{m}^{2}\right)$; Sol + MS1: solarization and metam sodium $\left(30.6 \mathrm{~g} / \mathrm{m}^{2}\right)$; Sol + MS1: solarization and metam sodium $\left(40.8 \mathrm{~g} / \mathrm{m}^{2}\right)$. Treatments with the same letter above columns are not significantly different for $\mathrm{p}=0.05($ Newmankeuls test). 
combinaisons sont les plus efficaces puisque $86 \%$ et $79 \%$ des Fusarium sont détruits, respectivement par Sol + MS2 et Sol + MS1. L'efficacité du metam sodium seul n'est que de $65 \%$ et celle de la solarisation de $57 \%$.

\section{Discussion}

Pour détecter les champignons, notamment Fusarium spp., il a fallu faire moins de dilutions pour les échantillons de sol pris plus en profondeur. Ceci prouve que la densité des Fusarium du sol diminue avec la profondeur. Ce résultat rejoint le constat fait par plusieurs auteurs qui ont rapporté que les Fusarium spp. sont très abondants entre 0 et $30 \mathrm{~cm}$ du profil de sol (Hamm et al., 2003). Sachant que la faible efficacité en profondeur est l'obstacle majeur qui rend le traitement du sol contre les formes de conservation du pathogène peu envisageable (Essarioui, 2006), la combinaison de plus d'une technique de désinfestation du sol est nécessaire.

Notre étude montre que la solarisation du sol durant les mois les plus chauds de l'année a un pouvoir important de suppression des champignons en général et des Fusarium spp. en particulier. Ces résultats confirment ceux obtenus précédemment (Essarioui, 2006), mais avec plus de réussite en profondeur. La nature du travail du sol peut être à l'origine de cette différence. Dans les essais menés dans les régions de Marrakech et Errachidia, le travail du sol avait consisté seulement en un covercropage superficiel, alors qu'ici les parcelles ont été labourées plus profondément, ce qui a pu permettre un transfert de chaleur plus important, se traduisant par une meilleure efficacité. Par ailleurs les conditions du milieu (nature du sol, température estivales, etc.) dans les différentes régions où nous avons testé la solarisation du sol (Marrakech, Errachidia et Zagora) peuvent aussi expliquer les différences de résultats. Nos résultats sont en concordance avec Porras et al. (2007) qui ont montré que l'élimination de Phytophtora cactorum peut atteindre $100 \%$ grâce à la solarisation du sol. Israel et al. (2005) ont aussi rapporté la possibilité de combattre Fusarium oxysporum f.sp. cumini par cette technique. Le rôle de la solarisation du sol a aussi été démontré par Tamietti et Valentino (2006) qui ont rapporté une réduction par la solarisation du sol de $58 \%$ à $96 \%$ des champignons telluriques dans les premiers $25 \mathrm{~cm}$ de profondeur. Les mêmes auteurs ont aussi avancé que la chaleur humide avait été à l'origine de la réduction des Fusarium spp. Récemment, plusieurs travaux ont rapporté le rôle de la solarisation du sol dans le contrôle de plusieurs fusarioses vasculaires (Gilardi et al., 2014; Yao et al., 2016; Kanaan et al., 2017). Cependant, l'utilisation de la seule technique de la solarisation se heurte au problème de sa faible efficacité en profondeur, ce qui limite son impact sur les germes de l'agent du bayoud les plus profonds.

Le metam sodium, à la dose prescrite du produit commercial utilisé $\left(61,2 \mathrm{~g} / \mathrm{m}^{2}\right)$, a montré une grande capacité à neutraliser les champignons du sol. Ce fumigant est un générateur de méthyle isothiocyanate qui a un large spectre d'action sur les pathogènes vivant dans le sol (Duniway, 2002; Xie et al., 2015). Mais une étude a montré que la génération et la dissipation du méthyle isothiocyanate sont très tributaires des conditions du sol (Shachaf et al., 2007). Cela suscite beaucoup de questions quant à la reproductibilité des succès si toutes les conditions ne sont pas réunies.
La combinaison des deux moyens, physique et chimique, est ici meilleure. L'association de la solarisation et de la fumigation à des doses basses a pu anéantir les germes des champignons telluriques jusqu'en profondeur. Ceci peut être apprécié de deux angles de vue différents. Le premier étant l'amélioration de l'efficacité de la solarisation en étendant l'effet du traitement à des niveaux rhizosphériques aussi bas que possible. Le deuxième est la diminution de l'intensité des dommages que des doses élevées de pesticides peuvent infliger à l'environnement. Ces résultats rejoignent ceux d'autres études. L'efficacité de la combinaison de la solarisation avec le metam sodium a été démontrée dans la lutte contre Fusarium oxysporum f. sp. radicis-lycopersici dans les serres de tomates (Antoniou et al., 2014). Eshel et al. (2000) ont aussi rapporté que la combinaison de la solarisation et du metam sodium à de faibles doses détruit à $100 \%$ Sclerotium rolfsii et Fusarium oxysporum f.sp. Basilici dans la couche $0-40 \mathrm{~cm}$ du sol. L'affaiblissement des agents pathogènes par des doses souslétales d'un agent de contrôle permettrait l'achèvement du travail par l'autre.

L'amélioration de l'efficacité en profondeur est aussi le résultat de l'extension de l'effet du metam sodium à travers le profil du sol grâce à sa combinaison avec la solarisation. Le metam sodium et ses dérivés étant peu mobiles dans le sol (Duniway, 2002), sa distribution homogène et verticale dans le sol pourrait être optimisée pas son association à la solarisation. En effet, DeVay (1998) a montré qu'au cours de la solarisation, l'humidité des différentes couches du sol connaît une fluctuation qui dépend de leur température. Cette dernière est douce durant la nuit et forte pendant les heures de forte insolation. Du fait de ces changements, le mouvement de l'eau à travers les différentes couches suit un rythme cyclique. Les radiations solaires induisent un flux descendant, et quand la surface du sol perd de la chaleur durant la nuit, l'eau remonte de nouveau. Ce phénomène serait aussi la cause de la réussite des traitements combinés: dans une parcelle traitée avec le metam sodium et couverte avec un film plastique, il y aurait un entraînement progressif du produit chimique en profondeur grâce au mouvement cyclique de l'eau entre le jour et la nuit. Le film plastique garde aussi les conditions de température et d'humidité nécessaires pour le bon fonctionnement du pesticide (Aharonson et Katan, 1991), lequel diffuserait verticalement, compensant ainsi la perte d'efficacité de la solarisation.

Une conséquence négative de la désinfestation du sol par solarisation, fumigation, ou la combinaison des deux, peut cependant être la destruction de la microflore bénéfique: champignons mycorhizes et agents de biocontrôle (Balakrishna et al., 2015). L'effet non-sélectif des moyens de désinfestation du sol crée alors un vide biologique susceptible d'être exploité par les germes résiduels de pathogènes recolonisant rapidement le sol. Dans le cas du palmier dattier, des mesures d'accompagnement post-traitement devraient être appliquées pour pérenniser l'effet du traitement et éviter la recolonisation du sol par une microflore indésirable. Par exemple, l'inoculation du sol, immédiatement après traitement, par des microorganismes bénéfiques, constitués de souches caractérisées et confirmées de mycorhizes et d'antagonistes vis-à-vis du pathogène, pourrait jouer un rôle fondamental dans le rétablissement d'une microflore bénéfique à même de protéger et d'améliorer la productivité des palmiers 
plantés sur des sols désinfectés. Ces microorganismes peuvent être introduits dans le sol sous forme de propagules mélangés avec des amendements organiques servant de sources nutritives et favorisant l'établissement, la croissance et le maintien de densités et d'activités microbiennes élevées.

En dépit de leur inapplicabilité dans les zones infestées à grande échelle, les résultats de notre étude montrent qu'en cas d'introduction de la maladie du bayoud dans les nouvelles plantations, un traitement localisé opportun, basé sur la combinaison de la solarisation avec la fumigation, pourrait éradiquer la maladie et prévenir sa progression dans ces zones. La conduite technique des vergers de palmier dans les aires d'extension, notamment la pratique de l'irrigation localisée ne permettant pas la dissémination du pathogène par les eaux d'irrigation abondante, et les mesures de prévention et d'exclusion rigoureuse pratiquées par les investisseurs, sont aussi des facteurs qui complètent cette stratégie de lutte. Ceci pourrait permettre de protéger les plantations contre l'effet dévastateur de la maladie et ainsi de sécuriser les investissements à long terme.

\section{Références}

Aharonson N, Katan J. 1991. Pesticide behavior in solarized and disinfected soils. In: Katan J, DeVay JE, ed. Soil solarization. Boca Raton, FL: CRC Press, pp. 131-138.

Antoniou PP, Tjamos EC, Giannakou JO. 2014. Low-cost and effective approaches of soil disinfestation of plastic house or open field crops in Greece. ISHS Acta Horticulturae 1044: VIII International Symposium on Chemical and Non-Chemical Soil and Substrate Disinfestation. DOI: 10.17660/ActaHortic.2014.1044.2.

Balakrishna AN, Lakshmipathy R, Bagyaraj DJ, Ashwin R. 2015. Effect of soil solarization on native AM fungi and microbial biomass. Agricultural Research 4 (2): 196-201.

Barakat RM, Al-Masri MI. 2012. Enhanced soil solarization against Fusarium oxysporum f. sp. lycopersici in the uplands. International Journal of Agronomy. DOI: 10.1155/2012/368654

DeVay JE. 1998. Historical review and principals of soil solarization. http://www.fao.org/docrep/t0455e/t0455e03.htm.

Duniway JM. 2002. Status of chemical alternatives to methyl bromide for pre-plant fumigation of soil. Phytopathology 92: 1337-1343.

Eshel D, Gamliel A, Grinstein A, Di Primo P, Katan J. 2000. Combined soil treatments and sequence of application in improving the control of soilborne pathogens. Phytopathology 90: 751-757.

Essarioui A. 2006. Comparaison entre les effets de la solarisation et du traitement chimique sur la microflore du sol de palmeraies et sélection d'un substrat organique inhibiteur du Fusarium oxysporum f.sp. albedinis agent causal du Bayoud du palmier dattier. Rapport de titularisation. Rabat, Maroc: Inra, 107 p.

Flood J. 2006. A review of Fusarium wilt of oil palm caused by Fusarium oxysporum f. sp. elaeidis. Phytopathology 96 (6): 660-662.

Funahashi F, Parke J. 2016. Effects of soil solarization and Trichoderma asperellum on Soilborne Inoculum of Phytophthora ramorum and Phytophthora pini in container nurseries. Plant Disease 100: 438-443.
Gilardi G, Demarchi S, Gullino ML, Garibaldi A. 2014. Effect of simulated soil solarization and organic amendments on fusarium wilt of rocket and basil under controlled conditions. J. Phytopath. 162 (9): 557-566.

Hamm PB, Ingham RE, Jaeger JR, Swanson WH, Volker KC. 2003. Soil fumigant effects on three genera of potential soilborne pathogenic fungi and their effect on potato yield in the Columbia Basin of Oregon. Plant Disease 87: 1449-1456.

Hwang S, Howard R, Strelkov S, Gossen B, Peng G. 2014. Management of clubroot (Plasmodiophora brassicae) on canola (Brassica napus) in western Canada. Canadian Journal of Plant Pathology 36: 49-65.

Israel S, Mawar R, Lpdha S. 2005. Soil solarisation, amendments and bio-control agents for the control of Macrophomina phaseolina and Fusarium oxysporum f. sp. cumini in aridisols. Annals of Applied Biology 146: 481-491.

Kanaan H, Medina S, Raviv M. 2017. The effects of soil solarization and compost on soil suppressiveness against Fusarium oxysporum f.sp. melonis. Compost Science \& Utilization. DOI: 10.1080/ 1065657X.2016.1277807.

Katan J. 1996. Soil solarization: integrated control aspects. In: Hall R, ed. Principles and practice of managing soilborne plant pathogens. St. Paul, MN: American Phytopathological Society Press, pp. 250-278.

Komada H. 1975. Developpement of a selective medium for quantitative isolation for Fusarium oxysporum from natural soil. Review of Plant Protection Research 8: 114-125.

Ploetz RC. 2015. Management of Fusarium wilt of banana: a review with special reference to tropical race 4. Crop Protection 73: 7-15.

Porras M, Barrau C, Arroyo FT, Santos B, Blanco C, Romero F. 2007. Reduction of Phytophthora cactorum in strawberry fields by Trichoderma spp. and soil solarization. Plant Disease 91:142-146.

Sedra MH. 1993. Caractères morphologiques et culturaux du Fusarium oxysporum f. sp. albedinis, agent causal de la fusariose vasculaire (Bayoud) du palmier dattier. Dakar, Sénégal: Séminaire sur les « interactions plantes-microorganismes ». 17-22/2/ 1992.

Sedra MH. 2005. Caractérisation des clones sélectionnés du palmier dattier pour combattre la maladie du Bayoud. Erfoud-Maroc: Actes $\mathrm{du}$ symposium international sur: le développement durable des systèmes oasiens. 08 au 10 mars 2005, pp. 72-79.

Shachaf TD, Austerweil M, Steiner B. 2007. Generation and dissipation of methyl isothiocyanate in soils following metam sodium fumigation: impact on Verticillium control and potato yield. Plant Disease 91: 497-503.

Tamietti G, Valentino D. 2006. Soil solarization as an ecological method for the control of Fusarium wilt of melon in Italy. Crop Protection 25: 389-397.

Xie H, Yan D, Mao L, Wang Q, Li Y, Ouyang C, et al. 2015. Evaluation of methyl bromide alternatives efficacy against soilborne pathogens, nematodes and soil microbial community. Plos One. doi: 10.1371/journal.pone.0117980.

Yao Y, Xue Z, Hong C, Zhu FX, Chen X, Wang W, et al. 2016. Efficiency of different solarization-based ecological soil treatments on the control of Fusarium wilt and their impacts on the soil microbial community. Applied Soil Ecology 108: 341-351.

Citation de l'article : Essarioui A, Sedra MH. 2017. Lutte contre la maladie du bayoud par solarisation et fumigation du sol. Une expérimentation dans les palmeraies du Maroc. Cah. Agric. 26: 45010. 